\title{
Pancreatitis aguda necrosante secundaria a hernia de hiato grado IV complicada: Presentación de un caso y revisión de la literatura
}

\author{
Acute necrotizing pancreatitis secondary to complicated grade IV \\ hiatal hernia: A case report and literature review

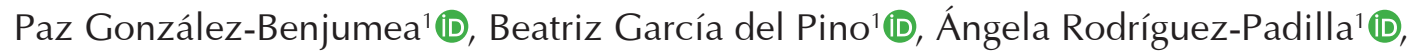 \\ Rafael Balongo-García ${ }^{2} \mathbb{D}$
}

1 Médica, especialista en Cirugía General y del Aparato Digestivo, Hospital Infanta Elena, Huelva, España.

2 Médico, especialista en Cirugía General y del Aparato Digestivo; jefe, Servicio de Cirugía General y del Aparato Digestivo, Hospital Juan Ramón Jiménez, Huelva, España.

\section{Resumen}

Introducción. En las hernias paraesofágicas tipo IV se produce la herniación del estómago junto a otros órganos abdominales. La herniación del páncreas es muy infrecuente.

Caso clínico. Varón de 57 años que acude por dolor torácico, disnea e intolerancia al decúbito. En la tomografía computarizada toracoabdominal se observa hernia diafragmática que contiene colon transverso, intestino delgado y páncreas, con reticulación de la grasa alrededor del mismo, compatible con pancreatitis aguda.

Conclusión. La asociación de hernia hiatal con páncreas herniado y pancreatitis es extremadamente infrecuente. El diagnóstico se estableció mediante tomografía computarizada y el tratamiento fue conservador, con cirugía diferida de la hernia de hiato.

Palabras clave. hernia hiatal; páncreas; pancreatitis; pancreatitis aguda necrotizante; cirugía; pancreatectomía.

\footnotetext{
Abstract

Introduction. In type IV paraesophageal hernias, the stomach is herniated along with other abdominal organs. Herniation of the pancreas is very rare.

Fecha de recibido: 09/02/2021 - Fecha de aceptación: 27/02/2021 - Publicación en línea: 17/09/2021

Correspondencia: Paz González-Benjumea, Hospital Infanta Elena, Calle Dr. Pedro Naranjo. Servicio de Cirugía General y del Aparato Digestivo. Teléfono: 656385074. Correo electrónico: paz.glez.bnj@gmail.com

Citar como: González-Benjumea P, García del Pino B, Rodríguez-Padilla A, Balongo-García R. Pancreatitis aguda necrosante secundaria a hernia de hiato grado IV complicada: Presentación de un caso y revisión de la literatura

Pancreatitis aguda necrosante secundaria a hernia de hiato grado IV complicada: Presentación de un caso y revisión de la literatura. Rev Colomb Cir. 2022;37:146-50. https://doi.org/10.30944/20117582.882

Este es un artículo de acceso abierto bajo una Licencia Creative Commons - BY-NC-ND https://creativecommons.org/licenses/by-nc-nd/4.0/deed.es
} 
Clinical case. A 57-year-old man presented with chest pain, dyspnea, and intolerance to decubitus. The thoracoabdominal computed tomography shows a diaphragmatic hernia containing the transverse colon, small intestine and pancreas, with reticulation of fat around it, compatible with acute pancreatitis.

Conclusion. The association of hiatal hernia with herniated pancreas and pancreatitis is extremely rare. The diagnosis was established by computerized tomography and the treatment was conservative, with delayed surgery for the hiatal hernia.

Keywords: hiatal hernia; pancreas; pancreatitis; acute necrotizing pancreatitis; surgery; pancreatectomy.

\section{Introducción}

Se conoce como hernia de hiato $(\mathrm{HH})$ la migración, transitoria o permanente, de al menos parte del estómago hacia la cavidad torácica, a través del hiato esofágico del diafragma ${ }^{1}$. Se clasifican en HH por deslizamiento o tipo I, que corresponde al $95 \%$ y se caracteriza por la herniación de la unión gastroesofágica, y $\mathrm{HH}$ paraesofágica (tipo II a IV), que representan el $5 \%^{2}$. La HH tipo IV es aquella en la que junto al estómago se produce la herniación de otros órganos abdominales. Es poco frecuente, representando el 5-7 \% de todas las HH paraesofágicas, siendo colon, intestino delgado, epiplón y bazo los órganos más comúnmente herniados ${ }^{3}$.

La herniación del páncreas está descrita en pocos casos reportados en la literatura, siendo aún menos frecuente la asociación con una pancreatitis aguda.

A continuación, presentamos el caso de un paciente con una pancreatitis aguda necrosante en el interior de una hernia del hiato y revisamos la literatura al respecto.

\section{Caso clínico}

Varón de 57 años, con antecedentes de obesidad mórbida e hipertensión sin tratamiento, que acude al Servicio de Urgencias por dolor torácico súbito e intenso, acompañado de disnea grado IV e intolerancia al decúbito. A la auscultación se aprecia una marcada disminución del murmullo vesicular en base derecha, con matidez a la percusión. La exploración abdominal era anodina.

Se solicitaron estudios de laboratorio urgentes con perfil abdominal, entre los que destacó un aumento de creatina-fosfocinasa (CPK), lipasa
(1380 mg/dL) y amilasa $(651 \mathrm{mg} / \mathrm{dL})$; y una radiografía de tórax, en la que se apreció hernia diafragmática derecha de gran tamaño. Se completó el estudio mediante tomografía computarizada (TC) toracoadominal, en la que se observó atelectasia de lóbulos medio e inferior derecho secundario a voluminosa hernia de hiato, con ocupación de gran parte del hemitórax derecho por colon transverso, mesocolon, asas de intestino delgado y cola de páncreas. Así mismo se apreció un aumento de densidad/reticulación de la grasa alrededor de la cabeza y cuerpo pancreático, compatible con pancreatitis aguda (Figura 1).

Ante la persistencia de la disnea intensa y ortopnea, a pesar de la descompresión con sonda nasogástrica, se decidió llevar a intervención quirúrgica urgente, practicándose laparotomía mediana supraumbilical, en la que se identificó gran hernia de hiato que contenía asas de intestino delgado, colon transverso, mesocolon, epiplón y cola de páncreas. Se redujo el contenido y se comprobó la viabilidad de las asas, apreciándose necrosis parcial del epiplón, que se reseca, y necrosis parcial del páncreas, para lo que se realizó pancreatectomía distal. Se terminó el procedimiento con hiatoplastia con malla no absorbible, guiada por sonda de Foucher de 36Fr, tras aproximación de pilares fijada con suturas.

Dada la inestabilidad hemodinámica del paciente durante toda la intervención, el postoperatorio inmediato cursó en la Unidad de Cuidados Intensivos, precisando terapia con líquidos endovenosos y drogas vasoactivas a dosis altas. A las 48 horas presentó fiebre, desarrolló neumonía por Klebsiella y posteriormente, a los 5 días de la intervención, infección de la herida quirúrgica por Enterobacter. Se procede a la extubación a los 


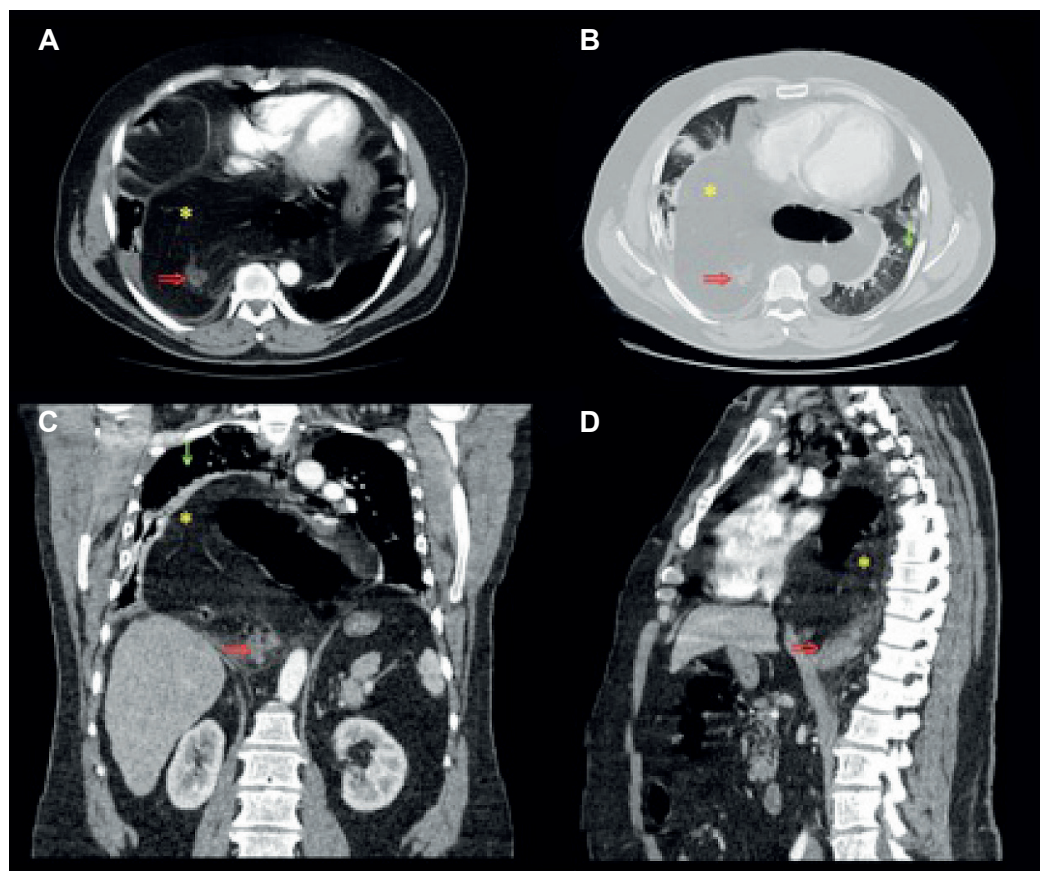

Figura 1. A y B: Cortes axiales. C: Corte coronal. D: Corte sagital. Imágenes de tomografía computarizada donde se observa cola de páncreas $(\Rightarrow)$ herniada en el interior de hernia hiatal $\left(^{*}\right)$ paraesofágica tipo IV, asociado a un aumento de densidad/reticulación de la grasa alrededor de la cabeza y cuerpo pancreático compatible con pancreatitis aguda. Además, se aprecia restricción pulmonar y atelectasias $(\vee)$.

15 días precisando BIPAP durante las 48 horas posteriores por insuficiencia cardiaca y atelectasias bibasales. Tras 21 días, pasa a planta de hospitalización, donde evoluciona favorablemente, permitiendo iniciar via oral y progresión de la dieta, precisando rehabilitación y curaciones locales de la herida quirúrgica, siendo dado de alta a su domicilio tras 30 días de hospitalización. La anatomía patológica de la pieza identificó una pancreatitis aguda necrosante.

\section{Discusión}

La herniación del páncreas está descrita en pocos casos en la literatura. En la mayoría de estos, es el cuerpo y la cola los que migran a través del diafragma, siendo la herniación de la cabeza pancreática un proceso extremadamente raro. Una revisión sistemática de los casos de páncreas herniados a través del hiato esofágico reportados desde 1958 hasta 2020 , identifica tan solo 17 pacientes diagnosticados de pancreatitis aguda secundaria a hernias paraesofágicas tipo IV ${ }^{1-18}$ (Tabla 1).

La pancreatitis aguda es un proceso inflamatorio del páncreas caracterizado por dolor abdominal y elevación de las enzimas pancreáticas, que puede progresar más allá del páncreas y generar fallo multiorgánico o incluso la muerte ${ }^{19}$. En cuanto a su etiología, la colelitiasis y el consumo excesivo de alcohol son las causas más frecuentes, siendo ambos responsables de aproximadamente el $80 \%$ de las pancreatitis agudas. Sin embargo, en un $15-25 \%$ de los casos, no es posible determinar la etiología, por lo que son catalogadas como pancreatitis agudas idiopáticas ${ }^{20}$.

Henkinbrant et al. ${ }^{21}$ postuló la hipótesis de que la pancreatitis aguda que se produce durante la migración del páncreas es a causa de traumatismos repetidos a medida que cruza la hernia, isquemia 
Tabla 1. Casos de pancreatitis aguda secundaria a herniación pancreática a través de hiato esofágico.

\begin{tabular}{|c|c|c|c|c|c|c|}
\hline Autor & Año & Edad & Sexo & $\begin{array}{c}\text { Área del páncreas } \\
\text { herniada }\end{array}$ & $\begin{array}{l}\text { Dilatación } \\
\text { Ducto }\end{array}$ & $\begin{array}{l}\text { Tratamiento } \\
\text { quirúrgico }\end{array}$ \\
\hline Kafka et al. ${ }^{4}$ & 1994 & ND & ND & Cabeza & ND & $\mathrm{Si}$ \\
\hline Chevallier et al. ${ }^{1}$ & 2001 & 70 & Hombre & Cuerpo y cola & ND & $\mathrm{Si}$ \\
\hline Laleman et al. ${ }^{5}$ & 2008 & 80 & Mujer & Páncreas completo & No & $\mathrm{Si}$ \\
\hline Rozas et al. ${ }^{6}$ & 2010 & 78 & Mujer & Cuerpo y cola & No & No \\
\hline Maksoud et al. ${ }^{7}$ & 2010 & 68 & Hombre & Cuerpo y cola & ND & $\mathrm{Si}$ \\
\hline Kumar et al. ${ }^{2}$ & 2013 & 89 & Hombre & Cuerpo & ND & No \\
\hline Boyce et al. ${ }^{8}$ & 2014 & 61 & Mujer & Cuello y cuerpo & $\mathrm{Si}$ & $\mathrm{Si}$ \\
\hline Lal et al. ${ }^{3}$ & 2015 & 70 & Mujer & ND & $\mathrm{Si}$ & No \\
\hline Lu et al. ${ }^{9}$ & 2015 & 88 & Hombre & Cuerpo y cola & No & No \\
\hline Patel et al. ${ }^{10}$ & 2016 & 65 & Hombre & Cuerpo y cola & ND & No \\
\hline Wang et al. ${ }^{11}$ & 2017 & 102 & Mujer & Cuerpo y cola & No & No \\
\hline Shafiq et al. ${ }^{12}$ & 2017 & 90 & Mujer & Cuerpo y cola & $\mathrm{Si}$ & No \\
\hline Do et al. ${ }^{13}$ & 2018 & 65 & Hombre & Cuerpo y cola & No & No \\
\hline Makhoul et al. ${ }^{14}$ & 2018 & 24 & Mujer & ND & No & $\mathrm{Si}$ \\
\hline Kamal et al. ${ }^{15}$ & 2019 & 79 & Mujer & Cuerpo y cola & $\mathrm{Si}$ & No \\
\hline Zackria et al. ${ }^{16}$ & 2019 & 33 & Hombre & Cuerpo & ND & $\mathrm{Si}$ \\
\hline Tomida et al. ${ }^{17}$ & 2020 & 86 & Mujer & Cuerpo y cola & $\mathrm{Si}$ & $\mathrm{Si}$ \\
\hline Nuestro caso & 2020 & 57 & Hombre & Cola & No & $\mathrm{Si}$ \\
\hline
\end{tabular}

*ND: No descrito

asociada con la tracción del pedículo vascular, u obstrucción intermitente del conducto pancreático principal.

El diagnostico de herniación pancreática se realiza mediante $\mathrm{TC}$, siendo el periodo sintomático el mejor momento, puesto que la reducción espontánea del contenido herniario es posible.

En cuanto al tratamiento, se debe optar por un manejo conservador de la pancreatitis aguda, siempre que esta no esté complicada, y cirugía diferida de la hernia de hiato.

Los estudios actuales demuestran que la reparación laparoscópica disminuye las complicaciones y la estancia hospitalaria ${ }^{22}$, por lo que se debería optar por esta vía de acceso siempre que sea posible.

\section{Conclusión}

La pancreatitis aguda en el seno de una hernia paraesofágica es una entidad muy infrecuente cuyo tratamiento debe ir dirigido hacia el manejo conservador inicial, con cirugía diferida siempre que la estabilidad del paciente lo permita. Sin embargo, en el caso presentado optamos por tratamiento quirúrgico urgente vía abierta ante los datos de hernia de hiato complicada con pancreatitis aguda en su interior, asociada a disnea grado IV, en un paciente que no tolera el decúbito debido a los síntomas compresivos de la misma.

\section{Cumplimientos de normas éticas}

Consentimiento informado: Para la publicación de este caso se obtuvo el consentimiento informado por parte del paciente.

Conflictos de interés: Los autores declararon no tener conflictos de interés en la elaboración del presente artículo.

Financiación: No se ha recibido ningún apoyo financiero para la realización del estudio.

\section{Contribución de los autores:}

Concepción y diseño del estudio: Paz González-Benjumea, Beatriz García del Pino, Ángela Rodríguez-Padilla, Rafael Balongo-García.

Adquisición de datos, análisis e interpretación de datos, redacción del manuscrito: Paz González-Benjumea, Beatriz García del Pino, Ángela Rodríguez-Padilla.

Revisión crítica: Rafael Balongo-García. 


\section{Referencias}

1. Chevallier P, Peten E, Pellegrino C, Souci J, Motamedi JP, Padovani B. Hiatal hernia with pancreatic volvulus: a rare cause of acute pancreatitis. Am J Roentgenol. 2001;177:373-4.

https://doi.org/10.2214/ajr.177.2.1770373

2. Kumar P, Turp M, Fellows S, Ellis J. Pancreatic herniation: a rare cause of acute pancreatitis? BMJ Case Rep. 2013:bcr2013201979. https://doi.org/10.1136/bcr-2013-201979

3. Lal A, Gupta P, Sinha SK. An unusual cause of abdominal pain in an elderly woman. Gastroenterology. 2015;148:E11-E12. https://doi.org/10.1053/j.gastro.2014.11.036

4. Kafka NJ, Leitman IM, Tromba J. Acute pancreatitis secondary to incarcerated paraesophageal hernia. Surgery. 1994;115:653-5.

5. Laleman W, Vanhoutte E, Vanbeckevoort D, Aerts R, Van Steenbergen W, Verslype C. A puzzling presentation of pancreatitis. Gut. 2008;57:1261-78. https://doi.org/10.1136/gut.2008.149401

6. González-Rozas M, Mendo-González M. A rare complication of hiatal hernia. Gastroenterology. 2010;139:E1E2. https://doi.org/10.1053/j.gastro.2009.12.069

7. Maksoud C, Shah AM, DePasquale J, Baddoura W, Spira R. Transient pancreatic hiatal herniation causing acute pancreatitis--a literature review. Hepatogastroenterology. 2010 ; 57:165-6.

8. Boyce K, Campbell W, Taylor M. Acute pancreatitis secondary to an incarcerated paraoesophageal hernia: A rare cause for a common problem. Clin Med Insights Case Rep. 2014;7:25-27. https://doi.org/10.4137/CCRep.S13079

9. Lu LX, Payne M, Theobald CN. Gastroenterology: Diaphragmatic herniation and pancreatitis. J Gastroenterol Hepatol. 2015;30:653-653. https://doi.org/10.1111/jgh.12880

10. Patel S, Shahzad G, Jawairia M, Subramani K, Viswanathan P, Mustacchia P. Hiatus Hernia: A Rare Cause of Acute Pancreatitis. Case Rep Med. 2016;2016:2531925. https://doi.org/10.1155/2016/2531925

11. Wang J, Thaker AM, El-Nachef WN, Watson RR. Transhiatal herniation of the pancreas: A rare cause of acute pancreatitis. ACG Case Rep J. 2017;4:e66. https://doi.org/10.14309/crj.2017.66
12. Shafiq M, Badshah MB, Badshah MB, Badshah MB, Watkins J. Pancreas herniation into the mediastinum: a case report. BMC Res Notes. 2017;10:450. https://doi.org/10.1186/s13104-017-2799-y

13. Do D, Mudroch S, Chen P, Prakash R, Krishnamurthy P. A rare case of pancreatitis from pancreatic herniation. J Med Cases. 2018;9:154-6. https://doi.org/10.14740/jmc3036w

14. Makhoul E, El Mir J, Loutfi T, Assaf Y. Hiatal hernia and acute pancreatitis. Int J Curr Res. 2018;10:68298-300.

15. Kamal MU, Baiomi A, Erfani M, Patel H. Rare sequalae of hiatal hernia causing pancreatitis and hepatitis: a case report. World J Gastrointest Endosc. 2019;11:249-55. https://doi.org/10.4253/wjge.v11.i3.249

16. Zackria R, Popa A. Transhiatal herniation as the cause of acute pancreatitis after Toupet fundoplication. ACG Case Reports Journal. 2019;6:e00156. https://doi.org/10.14309/crj.0000000000000156

17. Tomida H, Hayashi M, Hashimoto S. Massive hiatal hernia involving prolapse of the entire stomach and pancreas resulting in pancreatitis and bile duct dilatation: a case report. Surg Case Rep. 2020;6:11. https://doi.org/10.1186/s40792-020-0773-8

18. Moore TC. Esophageal hiatus hernia with obstructive incarceration of pylorus, pancreas, and duodenum. AMA Arch Surg. 1958;77:997-9. https://doi. org/10.1001/archsurg.1958.01290050167031

19. Nesvaderani M, Eslick GD, Vagg D, Faraj S, Cox MR. Epidemiology, aetiology and outcomes of acute pancreatitis: A retrospective cohort study. Int J Surg. 2015;23:68-74. https://doi.org/10.1016/j.ijsu.2015.07.701

20. Swaroop-Vege S. Etiology of acute pancreatitis. Walthman (MA): UpToDate; 2015. Fecha de consulta: 8 de marzo de 2017. Disponible en: http://www.uptodate.com

21. Henkinbrant A, Decoster O, Farchakh E, Khalek W. Acute pancreatitis caused by a voluminous umbilical hernia. Case report. Acta Gastroenterol Belg. 1989;52:441-7.

22. Correa J, Morales CH, Toro JP. Reparación laparoscópica de hernia hiatal gigante: técnica quirúrgica y seguimiento clínico, endoscópico y radiológico. Rev Colomb Cir. 2020;35:32-42. https://doi.org/10.30944/20117582.580 